\title{
Broadband x-ray properties of the gamma-ray binary 1FGL J1018.6-5856
}

An, Hongjun; Bellm, Eric; Bhalerao, Varun; Boggs, Steven E.; Christensen, Finn Erland; Craig, William W.; Fuerst, Felix; Hailey, Charles J.; Harrison, Fiona A.; Kaspi, Victoria M.

Published in:

Astrophysical Journal

Link to article, DOI:

$10.1088 / 0004-637 X / 806 / 2 / 166$

Publication date:

2015

Document Version

Publisher's PDF, also known as Version of record

Link back to DTU Orbit

Citation (APA):

An, H., Bellm, E., Bhalerao, V., Boggs, S. E., Christensen, F. E., Craig, W. W., Fuerst, F., Hailey, C. J., Harrison, F. A., \& Kaspi, V. M. (2015). Broadband x-ray properties of the gamma-ray binary 1FGL J1018.6-5856. Astrophysical Journal, 806(2), [166]. https://doi.org/10.1088/0004-637X/806/2/166

\section{General rights}

Copyright and moral rights for the publications made accessible in the public portal are retained by the authors and/or other copyright owners and it is a condition of accessing publications that users recognise and abide by the legal requirements associated with these rights.

- Users may download and print one copy of any publication from the public portal for the purpose of private study or research.

- You may not further distribute the material or use it for any profit-making activity or commercial gain

- You may freely distribute the URL identifying the publication in the public portal 


\title{
BROADBAND X-RAY PROPERTIES OF THE GAMMA-RAY BINARY 1FGL J1018.6-5856
}

\author{
Honguun An ${ }^{1,2}$, Eric Bellm ${ }^{3}$, Varun Bhalerao ${ }^{4}$, Steven E. Boggs ${ }^{5}$, Finn E. Christensen ${ }^{6}$, William W. Craig ${ }^{5,7}$, \\ Felix Fuerst $^{3}$, Charles J. Hailey ${ }^{8}$, Fiona A. Harrison ${ }^{3}$, Victoria M. Kaspi ${ }^{2,9}$, Lorenzo Natalucci ${ }^{10}$, Daniel Stern $^{11}$, \\ John A. TOMSICK ${ }^{5}$, AND William W. Zhang ${ }^{12}$ \\ ${ }^{1}$ Department of Physics/KIPAC, Stanford University, Stanford, CA 94305-4060, USA \\ ${ }^{2}$ Department of Physics, McGill University, Rutherford Physics Building, 3600 University Street, Montreal, QC H3A 2T8, Canada \\ ${ }^{3}$ Cahill Center for Astronomy and Astrophysics, California Institute of Technology, Pasadena, CA 91125, USA \\ ${ }^{4}$ Inter University Center for Astronomy and Astrophysics, Post Bag 4, Ganeshkhind, Pune 411007, India \\ ${ }^{5}$ Space Sciences Laboratory, University of California, Berkeley, CA 94720, USA \\ ${ }^{6}$ DTU Space, National Space Institute, Technical University of Denmark, Elektrovej 327, DK-2800 Lyngby, Denmark \\ ${ }^{7}$ Lawrence Livermore National Laboratory, Livermore, CA 94550, USA \\ ${ }^{8}$ Columbia Astrophysics Laboratory, Columbia University, New York, NY 10027, USA \\ ${ }^{9}$ McGill Space Institute, 3550 University Street, Montreal, QC H3A 2T8, Canada \\ ${ }^{10}$ Istituto Nazionale di Astrofisica, INAFIAPS, via del Fosso del Cavaliere, I-00133 Roma, Italy \\ 11 Jet Propulsion Laboratory, California Institute of Technology, Pasadena, CA 91109, USA \\ ${ }^{12}$ Goddard Space Flight Center, Greenbelt, MD 20771, USA \\ Received 2015 March 9; accepted 2015 May 7; published 2015 June 17
}

\begin{abstract}
We report on NuSTAR, XMM-Newton, and Swift observations of the gamma-ray binary 1FGL J1018.6-5856. We measure the orbital period to be $16.544 \pm 0.008$ days using Swift data spanning 1900 days. The orbital period is different from the 2011 gamma-ray measurement which was used in the previous X-ray study of An et al. using $\sim 400$ days of Swift data, but is consistent with a new gamma-ray solution reported in 2014 . The light curve folded on the new period is qualitatively similar to that reported previously, having a spike at phase 0 and broad sinusoidal modulation. The X-ray flux enhancement at phase 0 occurs more regularly in time than was previously suggested. A spiky structure at this phase seems to be a persistent feature, although there is some variability. Furthermore, we find that the source flux clearly correlates with the spectral hardness throughout all orbital phases, and that the broadband X-ray spectra measured with NuSTAR, XMM-Newton, and Swift are well fit with an unbroken powerlaw model. This spectrum suggests that the system may not be accretion-powered.
\end{abstract}

Key words: binaries: close - gamma rays: stars - stars: individual (1FGL J1018.6-5856) - X-rays: binaries

\section{INTRODUCTION}

Gamma-ray binaries are systems composed of a massive star and a compact object and from which persistent $\mathrm{GeV}$ and/ or $\mathrm{TeV}$ gamma-ray emission is detected and dominates the overall non-thermal spectrum. They emit across the electromagnetic spectrum from the radio to $\mathrm{TeV}$ gamma ray (see Mirabel 2012, for a review). There are only five gamma-ray binaries known to date (Dubus 2013), and only for one source has the compact object been identified (PSR B1259-63; Johnston et al. 1992).

Since most of the energy output of a gamma-ray binary is in the gamma-ray band, current theoretical studies focus on explaining the high energy emission properties. The gammaray emission models can be categorized into two classes: microquasar models (e.g., Romero et al. 2003; Bosch-Ramon \& Paredes 2004) and pulsar models (e.g., Tavani et al. 1994; Sierpowska-Bartoski \& Torres 2008). In the microquasar model, relativistic electrons in a jet generated close to the compact object Compton-upscatter the synchrotron emission of the jet itself and/or the stellar UV photons (e.g., KaufmanBernadó et al. 2002; Bosch-Ramon \& Paredes 2004), or relativistic hadrons collide with background nuclei creating pions that decay (e.g., Romero et al. 2003), producing gamma rays. In the pulsar model, pulsar wind particles are accelerated in the pulsar wind/stellar wind shock, and Compton-upscatter stellar photons to produce the observed gamma rays (e.g., Tavani et al. 1994; Tavani \& Arons 1997; Dubus 2006; Sierpowska-Bartoski \& Torres 2008).
Non-thermal X-ray emission in gamma-ray binaries is thought to be produced by the electrons which are accelerated in the pulsar wind/stellar wind shock (e.g., Tavani \& Arons 1997; Dubus 2006) or in relativistic jets formed close to the compact object (e.g., Bosch-Ramon \& Khangulyan 2009). The models predict varying $X$-ray fluxes and spectra depending on the properties of the shock, which are determined by the thrust of the winds and the orbital geometry of the binary system (e.g., Kaspi et al. 1995), or on the jet dynamics and cooling timescale (e.g., Bosch-Ramon \& Khangulyan 2009; Dubus et al. 2010). Hence, X-ray measurements can be used for constraining the orbital parameters and understanding the nature of the physical processes in gammaray binaries (see also Chernyakova et al. 2006; Takahashi et al. 2009; Takata et al. 2012).

The gamma-ray binary 1FGL J1018.6-5856 was discovered with Fermi in 2011. Ackermann et al. (2012) found modulation in the radio to gamma-ray bands with a period of $16.58 \pm 0.02$ days, identifying the source as a gamma-ray binary. They further identified the companion star to be an O6V(f) star. Soon after the discovery, subsequent broadband studies were carried out ( $\mathrm{Li}$ et al. 2011; Abramowski et al. 2012; An et al. 2013) in order to better characterize the source properties, but in no case were they able to identify the nature of the compact object.

X-ray properties of the gamma-ray binary 1FGL J1018.6-5856 were measured in detail with Swift. An et al. (2013) showed that the X-ray flux peak seen at phase 0 (gamma-ray maximum) by Ackermann et al. (2012) seems not 
Table 1

Summary of Observations used in this Work and Results of Spectral Analysis

\begin{tabular}{|c|c|c|c|c|c|c|c|c|}
\hline Observatory & Obs. ID & $\begin{array}{c}\text { Date } \\
\text { (MJD) }\end{array}$ & $\begin{array}{l}\text { Exposure } \\
(\mathrm{ks})\end{array}$ & $\phi$ & $\begin{array}{c}N_{\mathrm{H}} \\
\left(10^{22} \mathrm{~cm}^{-2}\right)\end{array}$ & $\Gamma$ & $\begin{array}{c}F_{3-10 \mathrm{keV}^{\mathrm{a}}} \\
\left(\mathrm{erg} \mathrm{s}^{-1} \mathrm{~cm}^{-2}\right)\end{array}$ & Mode \\
\hline$\overline{X M M}$ & 0604700101 & 55066 & $20 / 12$ & 0.6 & $0.65(5)$ & $1.65(7)$ & $5.1 \pm 0.2 \times 10^{-13}$ & $\overline{\mathrm{FW} / \mathrm{FW}^{\mathrm{b}}}$ \\
\hline$X M M$ & 0694390101 & 56302 & $104 / 73$ & 0.3 & $0.72(2)$ & $1.57(2)$ & $1.09 \pm 0.01 \times 10^{-12}$ & $\mathrm{FW} / \mathrm{SW}^{\mathrm{b}}$ \\
\hline Swift & $\begin{array}{c}00031912001- \\
00090191001\end{array}$ & $\begin{array}{c}55103- \\
56992\end{array}$ & 169 & $0.0-1.0$ & $0.72^{\mathrm{c}}$ & $1.2-1.8$ & $0.34-1.2 \times 10^{-12}$ & $\mathrm{PC}$ \\
\hline NuSTAR & 30002020002 & 56812 & 22 & 0.2 & $0.72^{\mathrm{c}}$ & $1.67(10)$ & $5.7 \pm 0.5 \times 10^{-13}$ & $\cdots$ \\
\hline NuSTAR & 30002020004 & 56862 & 23 & 0.2 & $0.72^{c}$ & $1.77(10)$ & $6.1 \pm 0.5 \times 10^{-13}$ & $\cdots$ \\
\hline NUSTAR & 30002020006 & 56911 & 25 & 0.2 & $0.72^{\mathrm{c}}$ & $1.64(8)$ & $7.8 \pm 0.5 \times 10^{-13}$ & $\ldots$ \\
\hline NUSTAR & 30002020008 & 56992 & 21 & 0.0 & $0.72^{\mathrm{c}}$ & $1.41(7)$ & $1.11 \pm 0.06 \times 10^{-12}$ & $\ldots$ \\
\hline
\end{tabular}

Notes.

a Absorption-corrected flux.

${ }^{\mathrm{b}}$ For MOS1,2/PN. FW: Full window. SW: small window.

${ }^{\mathrm{c}} N_{\mathrm{H}}$ was frozen for the Swift and NuSTAR data fit.

to be a persistent feature and instead shows a relatively large orbit-to-orbit variation. Furthermore, An et al. (2013) found evidence of a correlation between flux and spectral hardness in the X-ray band.

Recently, Coley et al. (2014) refined the gamma-ray period using Fermi observations with a longer baseline, and found the period to be $16.531 \pm 0.006$ days. ${ }^{13}$ Since this is slightly different from the value $(16.58 \pm 0.02$ days $)$ used for the previous X-ray study carried out by An et al. (2013), the X-ray results need to be refined using the new gamma-ray period. The baseline of the X-ray observations is long (5 years), and thus phases of later observations may change significantly.

Important questions to be addressed for gamma-ray binaries are: what is the nature of the compact object (known only for PSR B1259-63, Johnston et al. 1992), and what is the physical emission mechanism. If the source is powered by accretion, a complex continuum spectrum is expected whether the compact object is a neutron star or a black hole. Hence, accurate measurement of the spectrum will help us identify the compact object. Furthermore, searching for a spectral turn-over in the hard X-ray band (e.g., Grove et al. 1999; Coburn et al. 2002) and/or spectral lines often seen in high-mass X-ray binaries (HMXBs) may also provide clues about the emission mechanism of the source.

In this paper, we measure $\mathrm{X}$-ray properties of the gamma-ray binary 1FGL J1018.6-5856 more accurately than before using new observations taken with NuSTAR, Swift and with archival $X M M$-Newton observations. In Section 2, we describe the observations we used in this paper. We show data analysis and the results in Section 3. We then discuss our findings in Section 4, and conclude in Section 5.

\section{OBSERVATIONS}

We observed the gamma-ray binary 1FGL J1018.6-5856 with NuSTAR (Harrison et al. 2013) four times between 2014 June 4 and December 1 with exposures of $\sim 20 \mathrm{ks}$ for each observation. The total exposure was $90 \mathrm{ks}$. Soft X-ray band below and overlapping with the NuSTAR band (3-79 keV) was covered with Swift observations and two archival XMMNewton observations (see Table 1). The total exposure of the

\footnotetext{
$\overline{13}$ This value is from a private communication with J.B. Coley and is different from that in the abstract of Coley et al. (2014)
}

71 Swift observations was $169 \mathrm{ks}$, and each exposure was relatively short.

The NUSTAR observations were processed with the standard pipeline tools nupipeline and nuproducts of nustardas 1.4.1 integrated in HEASOFT 6.16. We used NuSTAR CALDB version 20140414 and applied the standard filters. ${ }^{14}$ In order to process the Swift data, we used the xrtpipeline tool along with HEASARC remote CALDB ${ }^{15}$ and standard filters (Capalbi et al. 2005). Note that the source was not clearly detected in some Swift observations, and that the Swift observations taken until MJD 55984 were reported previously (Ackermann et al. 2012; An et al. 2013). The XMM-Newton data were processed with epproc and emproc in Science Analysis System 14.0.0 $0^{16}$ using standard filters.

\section{DATA ANALYSIS AND RESULTS}

\subsection{Timing Analysis}

Detection of pulsations in gamma-ray binaries can be difficult for several reasons, such as the possibilities of an unfavorable emission geometry, absorption of soft X-rays by the wind, or a large background due to non-thermal unpulsed emission. Even in a favorable situation where the above effects are minimal, the Doppler effect due to binary motion can blur the pulse signal if the orbit is tight. For 1FGL J1018.6-5856, An et al. (2013) showed that the Doppler broadening is not a concern for a $20 \mathrm{ks}$ observation assuming a circular orbit with an inclination of $30^{\circ}$. We therefore attempt to search for the pulsation. Event arrival times measured at the spacecraft were transformed into those at the solar system barycenter with barycorr for the NUSTAR and barycen for the XMMNewton data. We did not search the Swift data because of the paucity of counts in individual Swift observations.

For the NUSTAR data, we produced an event list for each observation in the $3-20 \mathrm{keV}$ band using a circular aperture with $R=30^{\prime \prime}$. We performed the timing analysis with the data from each NUSTAR focal plane module ${ }^{17}$ as well as with the combined dataset. Above $20 \mathrm{keV}$ background dominates, and

\footnotetext{
${ }^{14}$ See http://heasarc.gsfc.nasa.gov/docs/nustar/analysis/nustar_swguide.pdf for more details.

$15 \mathrm{http}: / /$ heasarc.nasa.gov/docs/heasarc/caldb/caldb_remote_access.html

$16 \mathrm{http}: / / x m m . e s a c . e s a . i n t / s a s /$

${ }^{17}$ NUSTAR has two focal plane modules, FPMA and FPMB.
} 


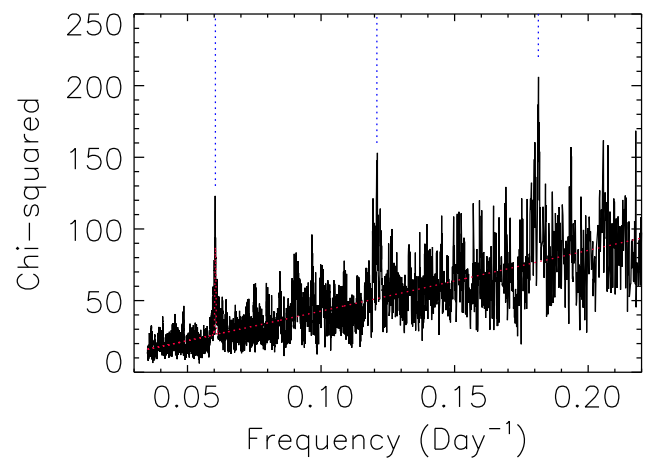

Figure 1. Chi-squared vs. frequency for the $0.5-10 \mathrm{keV}$ Swift X-ray data. The search step is 0.01 day and, the number of phase bins is 16 . Frequencies for the first three harmonics are denoted as blue dotted lines and the best-fit function is shown in red.

hence we adopt that as the high end of our band. Note that the results below do not depend strongly on the exact energy range or the aperture size. We folded the event time series to test periods between $10^{-4}-10^{3} \mathrm{~s}$, and calculated $Z_{1}^{2}$ (Buccheri et al. 1983). We find that $Z_{1}^{2}$ is fairly large for some test periods. However, we find that the large $Z_{1}^{2}$ seen in one observation is not reproduced in the others. We further verified that the large $Z_{1}^{2}$ values are not significant. Note that the measured $Z_{1}^{2}$ distribution does not follow a $\chi^{2}$ distribution, but has a long tail, and thus we used a functional distribution obtained by fitting the measured $Z_{1}^{2}$ distribution in order to estimate the significance. We performed the same study for the $X M M-N e w t o n / P N$ data in the $0.5-2$ and $0.5-10 \mathrm{keV}$ bands, and did not find any significant pulsations. Assuming the pulse profile is a sine function with a period in the range of $0.1-1 \mathrm{~s}$, we estimate the $90 \%$ upper limit for the pulse fractions to be $47 \%$ and $6 \%$ in the $3-20$ and $0.5-10 \mathrm{keV}$ bands, respectively.

Next, we refine the X-ray measurement of the orbital period by using a longer baseline using the Swift data over a longer time period than the previous work. Note that we did not use the data taken with XMM-Newton or NuSTAR because their count rate measurements cannot be directly compared to those of Swift. As was done by An et al. (2013), we use epoch folding (Leahy 1987) because of the unequal exposures of the observations. In the Swift observations, we extracted source and background events in the $0.5-10 \mathrm{keV}$ band within a $30^{\prime \prime}$ radius circle, and an annular region with inner radius $50^{\prime \prime}$ and outer radius $100^{\prime \prime}$, respectively. We then folded the event time series at test periods around $P_{\text {orb }}=16.531$ days (Coley et al. 2014), producing a light curve with 16 bins. We used the same epoch for phase 0 (MJD 55403.3) as that used in the previous studies (Ackermann et al. 2012; An et al. 2013). We calculated $\chi^{2}$ of the light curve for each trial period, and followed the fitting technique as described in Leahy (1987). Note, however, that we modeled the underlying continuum using a power-law function instead of the constant model employed by Leahy (1987), because the $\chi^{2}$ of the folded light curve is rising toward short periods (see Figure 1). The best-fit continuum model is $\chi_{\text {cont }}^{2} \sim P^{-1}$; the exact value of the power-law index varies between 0.9 and 1.1 depending on the fit range.

We find that the best-fit orbital period varies between 16.538 and 16.55 days, depending on the number of bins, and the search step or search range. We varied the number of bins between 8 and 18 to ensure that the light curve is resolved and the $\chi^{2}$ statistic is applicable, and the search step between 0.001 and 0.005 days, smaller than the uncertainty in the $P_{\text {orb }}$ measurement $\left(\Delta P_{\text {orb }} \lesssim 0.01\right.$ days $)$. The search range was varied between \pm 1 day and \pm 15 days. We find that the variations are within $1 \sigma$ of the measurements. The resulting period is $P_{\text {orb }}=16.544 \pm 0.008$ days. We show the folded light curve in Figure 2(a).

We also used the second harmonic to measure the orbital period, since it can be measured with better precision, and found that the measurement is more stable, varying only $6 \times 10^{-4}$ days as a function of the number of bins, search step or the search range. The result is $P_{\text {orb }}=16.543 \pm 0.004$ days. Our result is consistent with the Fermi measured value $\left(P_{\text {orb }}=16.531 \pm 0.006\right.$ days, Coley et al. 2014) with a null hypothesis probability $p=0.09$.

\subsection{Spectral Analysis}

Although $N_{\mathrm{H}}$ toward the source has been measured previously, the uncertainty was relatively large. Since there is one more XMM-Newton observation (Obs. ID 0694390101, Table 1) taken after the previous X-ray study (An et al. 2013), we can determine $N_{\mathrm{H}}$ more precisely using the XMM-Newton observations. We extracted the source spectrum from a circle with $R=16^{\prime \prime}$ (Obs. ID 0604700101) or $R=24^{\prime \prime}$ (Obs. ID 0694390101), and background spectra from a circle with $R=32^{\prime \prime}$ in a source-free region $\sim 200^{\prime \prime}$ vertically upwards along the detector column from the source. Note that we used different source extraction regions because of differences in exposure times.

Since it has been suggested that the spectral hardness varies orbitally we used different spectral slopes for observations taken at different phases (Figure 2). Thus, we fit the two $X M M-N e w t o n$ spectra separately allowing all the fit parameters to vary. We grouped the spectra to have 20 counts per bin, and fit them with an absorbed power-law model with the angr abundance in XSPEC (Anders \& Grevesse 1989) using $\chi^{2}$ statistics or $l$ statistics (Loredo et al. 1992). The two methods yield consistent results. The best-fit $N_{\mathrm{H}}$ values for the observations are statistically consistent with each other (Table 1). Best-fit $N_{\mathrm{H}}$ values obtained with a different abundance model (wilm in XSPEC; Willms et al. 2000) for the two spectra are still consistent with each other. Therefore, we use a common $N_{\mathrm{H}}$ value and find that a power-law model successfully explains the data (Figure 3, left). The best-fit value is $N_{\mathrm{H}}=7.2 \pm 0.2 \times 10^{21} \mathrm{~cm}^{-2}$, and we use this value throughout this paper. Note that using $N_{\mathrm{H}}=7.2 \pm 0.2 \times 10^{21} \mathrm{~cm}^{-2}$ does not change the other spectral parameters in Table 1 significantly. We find that using the wilm abundance model changes the best-fit $N_{\mathrm{H}}$ values (to $0.93 \pm 0.08 \times 10^{22} \mathrm{~cm}^{-2}$, $1.03 \pm 0.02 \times 10^{22} \mathrm{~cm}^{-2}$, and $1.02 \pm 0.02 \times 10^{22} \mathrm{~cm}^{-2}$ for Obs. IDs 0604700101, 0694390101, and combined, respectively), but the other spectral parameters do not change significantly. We note that the source count rates were less than $0.03-0.08 \mathrm{cps}$ for MOS $1 / 2$, and $0.1-0.3 \mathrm{cps}$ for $\mathrm{PN}$, and hence pile-up is not a concern. ${ }^{18}$

For the NUSTAR data, we extracted source and background events from circular regions with $R=30^{\prime \prime}$ and $R=45^{\prime \prime}$, respectively. Backgrounds were extracted in the same detector

\footnotetext{
${ }^{18} \mathrm{http}: / / \mathrm{xmm} 2$. esac.esa.int/docs/documents/CAL-TN-0200-1-0.pdf
} 

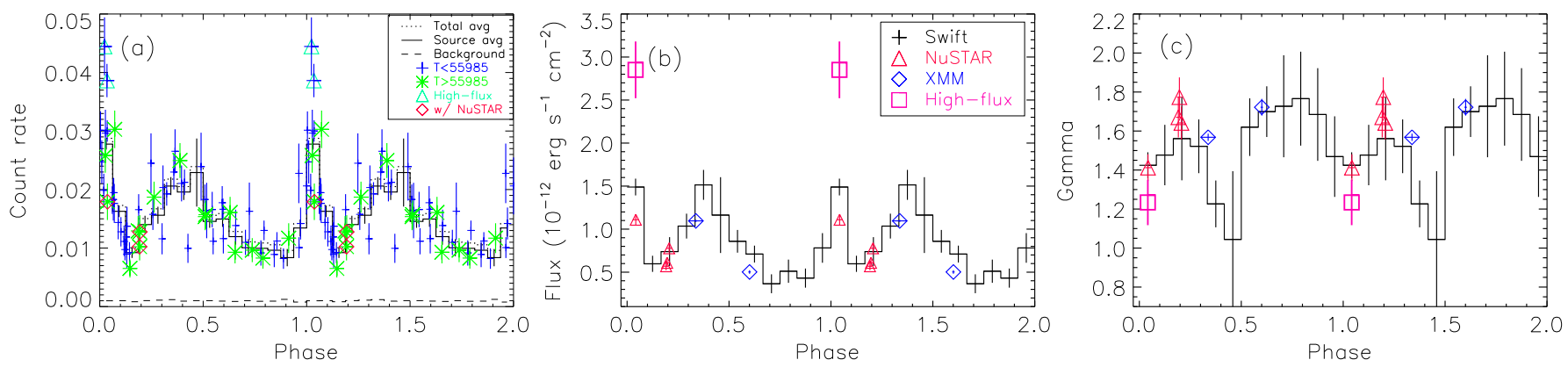

Figure 2. X-ray light curve and the best-fit spectral parameters for a power-law model. Only Swift data are shown in panel (a) and measurements made with all three instruments are shown in panels (b) and (c). (a) $0.5-10 \mathrm{keV}$ light curve as measured with Swift. Black dotted line shows the average count rate, black solid line is for the average source count rate, and the black dashed line shows the average background count rate. Blue data points are measurements reported by (before $55985 \mathrm{MJD}$ An et al. 2013), and green data points are new measurements (Coley et al. 2014, and this work). Cyan triangle denotes the "high-flux state," two observations which had significantly larger count rates than the others at the same phase, and red diamonds are for time periods in which NuSTAR observations were made. (b) 3-10 keV flux corrected for interstellar absorption. Data points for Swift, NuSTAR, and the XMM-Newton measurements are denoted in cross, triangle, and diamond, respectively. (c) The best-fit photon index. Same symbols as in (b) are used in (c).
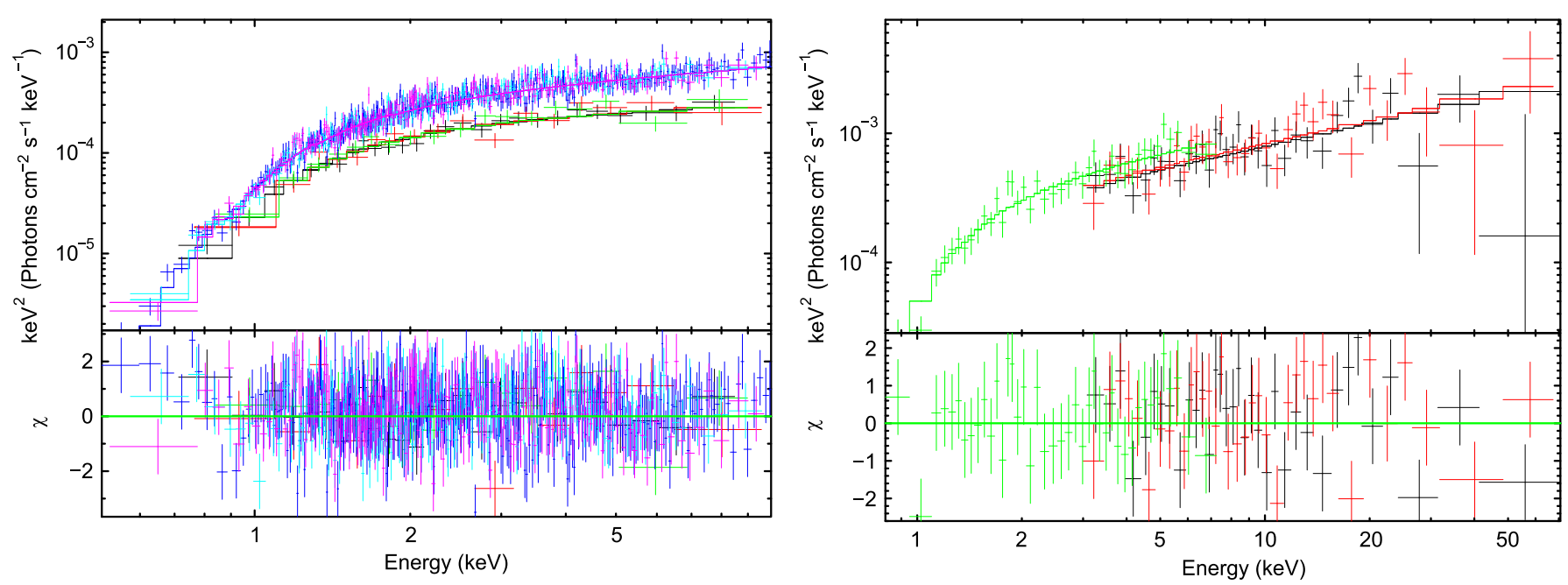

Figure 3. Broadband X-ray spectra obtained with XMM-Newton, NuSTAR and Swift. Left: XMM-Newton spectra obtained at phases 0.3 and 0.6. The harder and brighter spectra for phase 0.3 measured with PN, MOS1, and MOS2 are colored in blue, cyan, and magenta, respectively, and the spectra for phase 0.6 are colored in black, red, and green (see Figures 2(b) and (c)). Right: NuSTAR and Swift spectra for phase 0 without the high-flux state. Note that the Swift spectrum (green) is obtained by combining Swift observations taken at phase 0 excluding the high-flux state. Black and red data points show the NuSTAR spectra measured with FPMA and FPMB, respectively. The best-fit power-law model is shown in solid lines in each panel.

chip as the source, offset $\sim 4^{\prime}$ from the source region. The source was detected above the background up to $20-30 \mathrm{keV}$. We grouped the spectra to have a minimum of 20 counts per spectral bin, and used $\chi^{2}$ statistics and $l$ statistics; they provide consistent results.

We jointly fit the data with a power-law model having different photon indexes for different orbital phases, and found that the best-fit parameters are $\Gamma=1.69 \pm 0.05$ and $F_{3-10 \mathrm{keV}}=5.7 \pm 0.4 \times 10^{-13} \mathrm{erg} \mathrm{cm}^{-2} \mathrm{~s}^{-1}$ for $\phi=0.2$, and $\Gamma=1.41 \pm 0.07$ and $F_{3-10 \mathrm{keV}}=1.11 \pm 0.06 \times 10^{-12}$ erg cm${ }^{-2} \mathrm{~s}^{-1}$ for $\phi=0$. We find that a power-law model with a constant photon index across orbital phases can also explain the data if we let the flux vary between phases. However, a model with separate spectral indices for different phases provides a significantly better fit than one with constant phase-independent index throughout the phases, having an $F$-test probability that the improvement is just due to statistical chance of $2 \times 10^{-3}$. Using separate power-law indexes for the three observations taken at $\phi=0.2$ does not improve the fit. Furthermore, individual fits of the observations suggest that the photon index is statistically the same among the

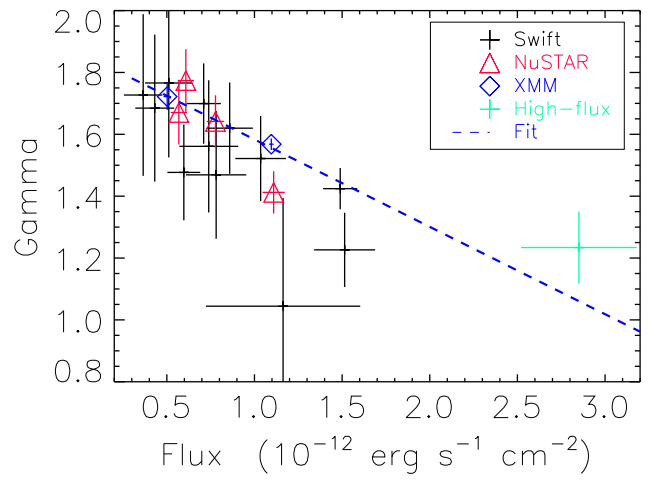

Figure 4. Photon index vs. $3-10 \mathrm{keV}$ flux. The dashed blue line shows the best-fit linear function.

observations taken at $\phi=0.2$ and that the photon index at phase 0 is different from that at phase 0.2 . We show the NuSTAR spectra in Figure 3 and the fit results in Figures 2 and 4.

For the Swift data, we extract the spectra using the same regions as for the timing analysis (Section 3.1). The center of 
the source extraction circle was determined for each observation separately. Since the source spectral properties vary with orbital phase (An et al. 2013), we performed phase-resolved spectroscopy. We folded the observations using the new timing solution we found in Section 3.1 and merged the data in each phase bin, for a total of twelve phase bins. We further produced two spectra for phase 0 , one for the high-flux state and another for the rest of the observations taken at that phase, and 11 spectra for the other phases hence producing a total of 13 spectra. We grouped the data to have 1 count per energy bin because of the paucity of counts in some phase bins, and used $l$ statistics. For the phases that have enough counts, we also tried to fit the spectra using $\chi^{2}$ statistics, after grouping to have more than 20 counts per energy bin, and found that the results are consistent with those obtained using $l$ statistics. We then fit all 13 spectra jointly with an absorbed power-law model with a common $N_{\mathrm{H}}$ (frozen at the XMM-Newton-measured value of $7.2 \pm 0.2 \times 10^{21} \mathrm{~cm}^{-2}$ ) throughout the observations but a separate photon index and flux for each spectrum. We find that the power-law model explains the data with photon indices of $\Gamma=1.2-1.8$ and $3-10 \mathrm{keV}$ fluxes of $F_{3-10 \mathrm{keV}}=0.34-$

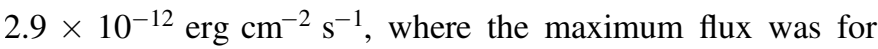
the high-flux state (see Figures 2(b) and (c)).

We also tried to determine $N_{\mathrm{H}}$ at each orbital phase using the Swift data. However, we were able to constrain the fit parameters reasonably only for phase 0 (without the high-flux state). We fit the spectrum for phase 0 (excluding the high-flux state) with a power-law model, and find that the best-fit parameters are $N_{\mathrm{H}}=7.7 \pm 1.2 \times 10^{21} \mathrm{~cm}^{-2}, \Gamma=1.4 \pm 0.1$, and $F_{3-10 \mathrm{keV}}=1.5 \pm 0.1 \times 10^{-12} \mathrm{erg} \mathrm{cm}^{-2} \mathrm{~s}^{-1}$. This $N_{\mathrm{H}}$ value at phase 0 is consistent with those obtained using the XMM-Newton data for phases 0.3 and 0.6 above, suggesting that $N_{\mathrm{H}}$ does not strongly vary as a function of orbital phase. Although we cannot clearly rule out orbital variation of $N_{\mathrm{H}}$, a $\sim 10 \%$ variation of $N_{\mathrm{H}}$ does not significantly change the Swift results.

Accretion-powered neutron star HMXBs often show spectral features such as emission lines or an exponential cutoff in the X-ray band (Coburn et al. 2002). We find that the X-ray spectrum of 1FGL J1018.6-5856 is well described with a power-law model without requiring any additional features (e.g., Figure 3). For example, fitting the spectrum for phase 0 with a cutoff power-law model ( $\mathrm{pow}^{\star}$ highecut in XSPEC) does not improve the fit, and the best-fit parameters are not constrained. We further changed the spectral grouping in order to have the spectra cover a broader energy range, and to see if a cutoff is required at higher energy. Specifically, we grouped the NuSTAR spectra to have more than 15 counts per energy bin, covering the $3-70 \mathrm{keV}$ band. We fit the spectra with a powerlaw model and a cutoff power-law model, and found the same results as above; no cutoff is required in the fit.

We performed additional analysis to determine the lower limit for the cutoff energy ( $E_{\text {cutoff }}$ of the highecut model). However, it is not possible to set a meaningful lower limit for $E_{\text {cutoff }}$ without constraining the e-folding energy $\left(E_{\mathrm{f}}\right.$ of the highecut model). We therefore limit $E_{\mathrm{f}}$ bewteen 6 and $12 \mathrm{keV}$, values obtained for a sample of accretion-powered neutron star HMXBs (Coburn et al. 2002), and found that the $90 \%$ lower limit for $E_{\text {cutoff }}$ is 39 and $34 \mathrm{keV}$ for $E_{\mathrm{f}}$ of 6 and $12 \mathrm{keV}$, respectively. Note that some accretion-powered black hole binaries are known to have the cutoff energy above
$70 \mathrm{keV}$ and that our data are not sensitive to such high energy cutoff.

\subsection{Spectral Variability}

The spectral hardness varies with orbital phase (Figure 2(c)) and flux (Figure 4). Figure 4 shows an apparent correlation between flux and spectral hardness. We fit the apparent correlation with a constant function and found that it does not provide an acceptable fit $\left(\chi^{2} /\right.$ degrees of freedom (dof) $=$ $55 / 18)$. We therefore added a linear slope to the constant function and find that the linear fit explains the data well $\chi^{2} /$ dof $=17 / 17$ ). The measured slope is $-0.28 \pm 0.04$ (per $10^{-12} \mathrm{erg} \mathrm{cm}^{-2} \mathrm{~s}^{-1}$ ), consistent with that reported by An et al. (2013). The best-fit function is shown in Figure 4.

An et al. (2013) suggested that there is evidence for a correlation between X-ray flux and spectral hardness. With the new and larger dataset, it is clear that the two quantities are correlated. For example, we find that the Spearman's rank order correlation coefficient is -0.84 and the significance is $5.6 \sigma$. We further verified that the X-ray flux and the photon index vary orbitally using the $\chi^{2}$ test, which resulted in $p<10^{-10}$ and $p \sim 10^{-5}$, respectively. Note that whether or not we include the high-flux data point in the correlation calculation does not significantly change the result.

Although the significance for the correlation is high, uncertainties in the measurements are significant (see Figure 4) and need to be considered for the significance calculation. In order to do so, we performed simulations. Note that the photon index and the flux are correlated in the spectral fit, and one needs to take into account the covariance. We do this by using the covariance matrices in the simulation as was done by An et al. (2013). In 100,000 simulations, a non-negative correlation occurred 316 times, which suggests that the significance of the negative correlation is $\sim 99.7 \%$. We also carried out simulations for the linear correlation, and measured the confidence level of the negative linear correlation to be $\sim 99.9 \%$.

We also checked for short-term variability $(\sim 10 \mathrm{ks})$ using the longer XMM-Newton observation (Obs. ID 0694390101) because it has the best statistics. We calculate the count rate and hardness ratio (ratio of count rates in two energy bands; e.g., $\left.C_{3}-10 \mathrm{keV} / C_{0.3-3 \mathrm{keV}}\right)$ on various time scales and energy bands. We find variabilities of $\sim 20 \%$ and $\sim 10 \%$ for the count rate and hardness ratio, respectively, but no correlation between them.

\section{DISCUSSION}

Our analysis of the NuSTAR, XMM-Newton, and Swift data is largely consistent with the X-ray results reported by An et al. (2013), but the current work provides improvements and refinements.

First, using longer Swift observations, we find that the orbital period of 1FGL J1018.6-5856 is $16.544 \pm 0.008$ days, consistent with the gamma-ray measurement $(16.531 \pm 0.006$ days; Coley et al. 2014). When folded on the new period, the light curve shows two distinct features; a spike at phase 0 and a broad sinusoidal hump (Figure 2), similar to those reported previously (Ackermann et al. 2012; An et al. 2013). With the new period measurement, however, we find that the spike at phase 0 is a persistent feature and shows less orbit-to-orbit variability than was suggested by An et al. (2013). Second, we clearly see the 
correlation between flux and spectral hardness for which An et al. (2013) found only marginal evidence. This is possible thanks to more sensitive observations made with NuSTAR, Swift and XMMNewton.

Note that we combined all the Swift observations taken over a period of $\sim 2000$ days for the spectral analysis. If there is long-term $\left(\gtrsim P_{\text {orb }}\right)$ and/or short-term (10-100 ks) variability, the combined results may be incorrect. This is a concern because there are only a few observations per orbital phase bin, and individual exposure of the observations is only $\sim$ ks. Furthermore, if the orbital period is not accurate or varies with time, phases of later observations will change, introducing an additional error to the analysis. However, the agreement of the Swift measurements with the NuSTAR and XMM-Newton results suggests that the errors may not be large compared to the statistical uncertainties, having no significant impact on the results. For example, the significance of the correlation between flux and photon index is still $\gtrsim 99 \%$ when adding $10 \%$ systematic uncertainty to the Swift measurements.

The broadband X-ray spectra of 1FGL J1018.6-5856 at phases 0 and 0.2 are well described with a power-law model in the $0.5-40 \mathrm{keV}$ band. Recently, Waisberg \& Romani (2015) find that, based on parameter space consistent with radial velocity measurements, a neutron star model is preferred over a typical stellar mass black hole, although both classes are still allowed for 1FGL J1018.6-5856. We check to see if the source shows any evidence for accretion, such as line features or an exponential cutoff in the X-ray spectrum, as is often seen in neutron star HMXBs, and find none (Figure 3). Furthermore, we find no clear evidence for an exponential cutoff at $E_{\text {cutoff }}<70 \mathrm{keV}$ (for spectrum at phase 0). We, therefore, set the $90 \%$ lower limit for $E_{\text {cutoff }}$ to be $34-39 \mathrm{keV}$ for e-folding energies of 6-12 keV ( $E_{\mathrm{f}}$; see Coburn et al. 2002, for the range of $E_{\mathrm{f}}$ of neutron star HMXBs). This lower limit is large for a neutron star HMXB (typical $E_{\text {cutoff }} \sim 10-20 \mathrm{keV}$; e.g., see Coburn et al. 2002). Note that the X-ray pulsar X Per (also known as 4U 0352+309) for which Coburn et al. (2002) did not find a clear spectral cutoff turned out to have a cutoff at $69 \mathrm{keV}$ (Lutovinov et al. 2012), which is comparable to the energy under which we did not find any evidence for a spectral cutoff in 1FGL J1018.6-5856. Also, high cutoff energies $\gtrsim 70 \mathrm{keV}$ have been seen in black hole binaries (Grove et al. 1999). Therefore, we cannot clearly rule out the possibility that 1FGL J1018.6-5856 is a black hole binary or a neutron star bianry with unusually high cutoff energy based only on the spectral cutoff. Nevertheless, the continuum spectrum of X Per or other X-ray binaries is very complex (e.g., Coburn et al. 2002) while we see a simple power-law spectrum for 1FGL J1018.6-5856. This suggests that 1FGL J1018.6-5856 may be a non-accreting neutron star system, which has also been suggested for another gamma-ray binary LS 5039 (e.g., Torres et al. 2011).

In analogy to LS 5039, we may identify the location of the sinusoidal X-ray peak at $\phi \sim 0.4$ (Figure 2) as inferior conjunction, and the gamma-ray peak at $\phi \sim 0$ (Coley et al. 2014) as superior conjunction (Abdo et al. 2009a; Kishishita et al. 2009). Then, the phase difference of $\Delta \phi \sim 0.4$ between the two conjunctions implies that the orbit is eccentric. We note, however, that it is not clear whether the X-ray and gamma-ray peaks are physically related to the conjunctions or the apastron/periastron passages, and that alignment of the $\mathrm{X}$-ray and gamma-ray peaks with inferior and superior conjunctions may not be precise. Therefore, more observations and detailed modeling are required in order to draw a firm conclusion.

We find that the X-ray spectral properties of 1FGL J1018.6-5856 clearly show orbital modulation. Pulsar models for gamma-ray binaries often attribute such orbital modulation with orbital variation in the adiabatic cooling timescale (Khangulyan et al. 2007, 2008), the electron injection spectrum, or the location and the shape of the wind nebula (Dubus 2006). The pulsar models have been applied to the similar system LS 5039 (e.g., spectral variability and recurring X-ray flares; Kishishita et al. 2009; An et al. 2013), and have reproduced the overall spectral energy distribution (e.g., Dubus 2006). However, whether or not these models can explain the spiky feature at phase 0 we see in 1FGL J1018.6-5856 needs to be investigated.

We note that the high-flux state observed with Swift at phase 0 (Figures 2(a) and (b)) is not reproduced in other observations taken at the same phase. It may be because the two observations in the high-flux state were made in a very narrow phase interval and the later observations did not cover that phase interval. In order to see if this is the case, we first verified that the high-flux state was not produced by short timescale variability $(\sim \mathrm{ks})$; it lasted for the full duration of the exposures of the observations $(24 \mathrm{ks}$ at MJD 55585.7 and $7 \mathrm{ks}$ at MJD 55618.7 for Obs. IDs 00031912004 and 00031912011 , respectively) which cover a phase interval of $\Delta \phi=0.022$ (at $\phi=0.034 \pm 0.011$ for $P_{\text {orb }}=16.54$ days $)$. We then measured the phases of the other observations. We find that there are four observations made at the high-flux phase interval, and none of them was in the high-flux state. Since the phase of an observation can change significantly for a different orbital period, we further varied $P_{\text {orb }}$ within the measurement uncertainty of 0.01 day, and find the same result. This suggests that there is orbit-to-orbit flux variability at phase 0 .

We find that the duration of the high-flux state is longer than $24 \mathrm{ks}$ and shorter than 1.8 days. The minimum duration is set to be $24 \mathrm{ks}$ because the high-flux states last during the observation (see above). The maximum duration is set to be the interval between a high-flux state and the next non-highflux observation, which is 1.8 days for both high-flux states. As noted by An et al. (2013), the observational properties of the flare such as duration and orbital repeatability look more like that of LS 5039 (e.g., Kishishita et al. 2009) than those of LS I +61³03 (e.g., Smith et al. 2009; Li et al. 2011). This may support the idea that the flares are produced by clumpiness of the stellar wind (e.g., Zdziarski et al. 2010) since the stellar companion (Be star) of LS I $+61^{\circ} 303$ is different from those (O stars) of 1FGL J1018.6-5856 and LS 5039 as the flare properties do. However, how the clumpiness produces flares at one orbital phase for 1FGL J1018.6-5856 or LS 5039 but not at random orbital phases needs to be further investigated.

\section{CONCLUSIONS}

We present results of NuSTAR, Swift, and XMM-Newton observations of the gamma-ray binary 1FGL J1018.6-5856. Using the Swift data, we measured the orbital period of the source to be $16.544 \pm 0.008$ days, in agreement with the refined gamma-ray measurement of Coley et al. (2014). The new period is only slightly different from that used in our previous X-ray study, and hence our spectral and temporal 
analysis results agree well with the previous X-ray measurements. We find that the flux enhancement at phase 0 occurs more regularly in time than was suggested previously based on Swift data. The new NUSTAR and XMM-Newton data allow us to show clearly the correlation between X-ray flux and spectral hardness of 1FGL J1018.6-5856. Finally, the broadband X-ray spectrum of 1FGL J1018.6-5856 suggests that it may not be an accretion-powered system.

We thank R. W. Romani for useful discussions. This work was supported under NASA Contract No. NNG08FD60C, and made use of data from the NUSTAR mission, a project led by the California Institute of Technology, managed by the Jet Propulsion Laboratory, and funded by the National Aeronautics and Space Administration. We thank the NuSTAR Operations, Software and Calibration teams for support with the execution and analysis of these observations. This research has made use of the NUSTAR Data Analysis Software (NuSTARDAS) jointly developed by the ASI Science Data Center (ASDC, Italy) and the California Institute of Technology (USA). This research has made use of data obtained from the High Energy Astrophysics Science Archive Research Center (HEASARC), provided by NASA's Goddard Space Flight Center. H.A. acknowledges supports provided by the NASA sponsored Fermi Contract NAS5-00147 and by Kavli Institute for Particle Astrophysics and Cosmology (KIPAC). LN wishes to acknowledge the Italian Space Agency (ASI) for financial support by ASI/INAF grant I/037/12/0-011/13.

\section{REFERENCES}

Abdo, A. A., Ackermann, M., Ajello, M., et al. 2009a, ApJL, 706, L56 Abramowski, A., Acero, F., Aharonian, F., et al. 2012, A\&A, 541, A5 Ackermann, M., Ajello, M., Ballet, J., et al. 2012, Sci, 335, 189 An, H., Dufour, F., Kaspi, V. M., \& Harrison, F. A. 2013, ApJ, 775, 135 Anders, E., \& Grevesse, N. 1989, GeCoA, 53, 197 Bosch-Ramon, V., \& Paredes, J. M. 2004, A\&A, 417, 1075
Bosch-Ramon, V., \& Khangulyan, D. 2009, IJMPD, 18, 347

Buccheri, R., Bennett, K., Bignami, et al. 1983, A\&A, 128, 245

Capalbi, M., Perri, M., Saija, B., Tamburelli, F., \& Angelini, L. 2005, The Swift XRT Data Reduction Guide, Tech. Rep. 1.2

Chernyakova, M., Neronov, A., \& Walter, R. 2006, MNRAS, 372, 1585

Coley, J. B., Corbet, R. H. D., Cheung, C. C., et al. 2014, AAS Meeting Abstracts, 14, 122.10

Coburn, W., Heindl, W. A., Rothschild, R. E., et al. 2002, ApJ, 580, 394

Dubus, G. 2006, A\&A, 456, 801

Dubus, G. 2013, A\&ARv, 21, 64

Dubus, G., Cerutti, B., \& Henri, G. 2010, MNRAS, 404, L55

Grove, J. E., Johnson, W. N., Kroeger, R. A., et al. 1999, ApJ, 500, 899

Harrison, F. A., Craig, W. W., Christensen, F. E., et al. 2013, ApJ, 770, 103

Johnston, S., Manchester, R. N., Lyne, A. G., et al. 1992, ApJL, 387, L37

Kaspi, V. M., Tavani, M., Nagase, F., et al. 1995, ApJ, 453, 424

Kaufman-Bernadó, M. M., Romero, G. E., \& Mirabel, I. F. 2002, A\&A, 385, L10

Khangulyan, D., Hnatic, S., Aharonian, F., \& Bogovalov, S. 2007, MNRAS, 380,320

Khangulyan, D. V., Aharonian, F. A., Bogovalov, S. V., Koldoba, A. V., \& Ustyugova, G. V. 2008, IJMPD, 17, 1909

Kishishita, T., Tanaka, T., Uchiyama, Y., \& Takahashi, T. 2009, ApJL, 697, L1 Leahy, D. A. 1987, A\&A, 180, 275

Li, J., Torres, D. F., Chen, Y., et al. 2011, ApJL, 738, L31

Li, J., Torres, D. F., Zhang, S., et al. 2011, ApJ, 733, 89

Loredo, T. J. 1992, in Statistical Challenges in Modern Astronomy, ed. E. D. Feigelson, \& G. J. Babu (New York: Springer), 275

Lutovinov, A., Tsygankov, S., \& Chernyakova, M. 2012, MNRAS, 423 1978

Mirabel, I. F. 2012, Sci, 335, 13

Romero, G. E., Torres, D. F., Kaufman-Bernadó, M. M., \& Mirabel, I. F. 2003, A\&A, 410, L1

Sierpowska-Bartoski, A., \& Torres, D. F. 2008, APh, 20, 239

Smith, A., Kaaret, P., Holder, J., et al. 2009, ApJ, 693, 1621

Takahashi, T., Kishishita, T., Uchiyama, Y., et al. 2009, ApJ, 697, 592

Takata, J., Okazaki, A. T., Nagataki, S., et al. 2012, ApJ, 750, 70

Tavani, M., Arons, J., \& Kaspi, V. M. 1994, ApJL, 433, L37

Tavani, M., \& Arons, J. 1997, ApJ, 477, 439

Torres, D. F. 2011, in Proc. First Session of the Sant Cugat Forum on Astrophysics 532, High-Energy Emission from Pulsars and their Systems, ed. N. Rea, \& D. F. Torres (Berlin: Springer)

Waisberg, I., \& Romani, R. W. 2015, ApJ, 805, 18

Willms, J., Allen, A., \& McCray, R. 2000, ApJ, 542, 914

Zdziarski, A. A., Neronov, A., \& Chernyakova, M. 2010, MNRAS, 403, 1873 